\title{
The Cornea in Sjögren's Syndrome: An In Vivo Confocal Study
}

\author{
Edoardo Villani, Daniela Galimberti, Francesco Viola, Chiara Mapelli, and \\ Roberto Ratiglia
}

Purpose. To analyze the in vivo morphology of corneal cells and nerves in dry eye associated with primary (SSI) and secondary (SSII) Sjögren's syndrome and to study its relationship with the clinical evaluation.

Methods. Thirty-five patients with SS and 20 age- and gendermatched control subjects were studied. Confocal microscopy was used to investigate corneal thickness, epithelial and stromal cellular density, and subbasal plexus morphology.

Results. Corneal central thickness was $514.74 \pm 19.85 \mu \mathrm{m}$ in the SS group and $550 \pm 21.46 \mu \mathrm{m}$ in the control group $(P<$ $0.0001, t$-test); stromal central thickness was $456.62 \pm 18.05$ $\mu \mathrm{m}$ in the SS group and $487.35 \pm 20.40 \mu \mathrm{m}$ in the control group $(P<0.0001)$. The density of the superficial epithelial cells in the SSI and SSII groups was $965.40 \pm 96.00$ and $999.80 \pm 115.67$ cells $/ \mathrm{mm}^{2}$, respectively, and $1488.55 \pm$ 133.74 cells $/ \mathrm{mm}^{2}$ in the control group $(P<0.001$, ANOVA). The number of subbasal nerves was $3.34 \pm 0.76$ in the SS group and $5.10 \pm 0.79$ in the control group $(P<0.0001$, $t$-test). The average grade of nerve tortuosity was $2.62 \pm 0.94$ in the SS group and $1.20 \pm 0.70$ in the control group $(P<$ 0.0001). Statistically significant correlations were found between clinical data and confocal microscopy data.

Conclusions. Corneal thickness, cells, and nerves show morphologic changes in patients with dry eye associated with SS. The in vivo confocal study of these alterations may be important in better understanding the complexity of the ocular surface morphofunctional unit and the potentials of therapeutic approaches for the control of the phlogistic process and neuroprotection. (Invest Ophthalmol Vis Sci. 2007;48: 2017-2022) DOI:10.1167/iovs.06-1129

G jögren's syndrome (SS) is a chronic inflammatory disease

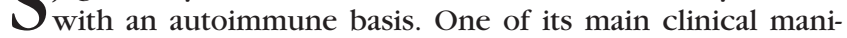
festations is keratoconjunctivitis sicca (KCS). In primary SS (SSI), the presence of specific antibodies and signs of the infiltration of mononuclear cells in the exocrine glands accompany reduced tear and saliva secretion. In secondary SS (SSII), the typical symptoms of the primary form are coupled by

From the Clinica Oculistica Università degli Studi di Milano, Fondazione Ospedale Maggiore Policlinico, Mangiagalli e Regina Elena, IRCCS (Istituti di Ricovero e Cura a Carattere Scientifico), Milan, Italy.

Presented at the 78th annual meeting of the Association for Research in Vision and Ophthalmology, Fort Lauderdale, Florida, May, 2006

Submitted for publication September 20, 2006; revised November 7, 2006; accepted February 9, 2007.

Disclosure: E. Villani, None; D. Galimberti, None; F. Viola, None; C. Mapelli, None; R. Ratiglia, None

The publication costs of this article were defrayed in part by page charge payment. This article must therefore be marked "advertisement" in accordance with 18 U.S.C. $\$ 1734$ solely to indicate this fact.

Corresponding author: Edoardo Villani, Clinica Oculistica Università degli Studi di Milano, Fondazione Ospedale Maggiore Policlinico, Mangiagalli e Regina Elena, IRCCS, Via Francesco Sforza 35, 20122 Milan, Italy; eddy.villani@tiscali.it. another well-defined autoimmune disorder (e.g., rheumatoid arthritis, SLE [systemic lupus erythematosus], scleroderma). ${ }^{1-3}$

For many years, KCS was considered to be the result of a reduced secretion of the aqueous component in tears. Today, the ocular surface is considered to be a morphofunctional unit comprising lachrymal film, cornea, limbus, conjunctiva, mucoepidermal junction, accessory lachrymal glands, Meibomian glands, principal lachrymal gland, and nervous plexus. The new interpretation affirms the concept that the alteration of one of the individual components of the ocular surface system is followed by pathologic events that extend to the other structures. ${ }^{4-6}$

Experimental and clinical studies hypothesized that, in Sjögren's syndrome, KCS may be a primitive disease of the ocular structure, only partly dependent on the deficit of lachrymal secretions and therefore susceptible to an evolution of its own. Alterations in the epithelium surfaces seem too important to be explained simply by a reduction in lachrymal secretions for a primitive compromising of the producing gland. Surface morphologic changes, including the presence of inflammatory cells and the expression of histocompatibility antigens (HLA-DR), suggest the participation of epithelial cells in an important inflammatory and immunologic process in the pathogenesis of KCS associated with SS. Furthermore, SS implies an alteration in the epithelium and subepithelium innervations, with consequent reduction in corneal sensitivity. An intact corneal innervation has a trophic effect on the epithelial cells and is responsible for normal blinking and lachrymal reflex. Considering that the ocular surface, lachrymal glands, and nerve endings form a functional unit, the compromising of one point in this reflex arc could lead to disease of the ocular surface. It is necessary to study the modifications of the corneal surface further in depth, for better understanding of the physiopathology of KCS associated with SS, for better differentiation from the degenerative form, and for fine-tuning a more precise and correct therapeutic treatment.

Traditional methodological clinical and instrumental diagnostics are unsatisfactory and limited in the semiological and functional study of KCS. The use of in vivo confocal microscopy permits a completely new approach in the study of the microscopic morphology of the cornea, offering a resolution comparable to histologic examination but with an analysis that is in no way invasive and is quickly executed, safe, and repeatable. Furthermore, it allows sequential images to be obtained of corneal diseases in evolution.

\section{Methods}

\section{Patients}

Fifteen of the patients (12 women and 3 men) recruited had SSI (average age, $52.3 \pm 10.3$ years; range, $39-73$ ), and 20 patients (14 women and 6 men) had SSII with (average age, $56.5 \pm 16.4$ years; range, $30-74)$. The patients came to our attention at the Ocular Immunopathology Division after being sent for a visit by the Rheumatology Clinic, where a diagnosis of SSI or SSII was made according to the criteria proposed by the American-European Consensus Group. ${ }^{7}$ 
The control group (C) was formed by 20 healthy people (13 women and 7 men; average age, $51.2 \pm 18.2$ years; range, $27-76$ ), affiliated with our General Clinic.

Exclusion criteria were the presence of lymphoma, AIDS, sarcoidosis, or diabetes mellitus; corneal dystrophy and inflammation; systemic therapy with pharmaceutical drugs with known corneal toxicity; topical therapy with antiglaucoma drugs, steroids, or NSAIDs; the use of contact lenses; and previous interventions of ophthalmic surgery.

Each subject had both eyes examined, and the data from the eye obtaining the highest score in the Van Bijsterveld test with fluorescein were used for statistical analysis. In the case of subjects who had the same parameters in both eyes, the selection criteria were on the order of minor corneal sensitivity, evaluated using the Cochet-Bonnet esthesiometer and the lowest result in the Schirmer I test.

All the participants in the study gave informed consent, compiled in accordance with the Italian laws regarding privacy. The study was performed in compliance with the tenets of the Declaration of Helsinki.

\section{Clinical Evaluation}

A careful case history was compiled for each participant, and each person completed a questionnaire (Ocular Surface Disease Index; OSDI ${ }^{8}$ for a standardized evaluation of the symptomatology of dry eye. Besides considering the frequency of dry eye symptoms, the questionnaire attempts to rate their intensity by investigating their ability to interfere with the participant's daily activities.

The participants in the study underwent an ophthalmic examination that included biomicroscopic examination of the ocular adnexa and anterior segment, evaluation of corneal sensitivity by using the Cochet-Bonnet nylon-thread esthesiometer, a Schirmer I test under topical anesthesia, staining with sodium fluorescein $1 \%$ collyrium (evaluated using the van Bijsterveld method), measurement of the breakup time of the lachrymal film (BUT), and measurement of the IOP with a Goldmann applanation tonometer.

\section{Confocal Microscopy}

Image Acquisition. The confocal microscopic examination (Confoscan 2.0; Nidek Technologies, Vigonza, Italy) was performed at the level of the corneal apex. A drop of anesthetic (oxybuprocaine chloride $0.4 \%$ ) was instilled in the lower conjunctival fornix before examination. During the test, the object lens of the microscope was covered with gel (hydroxypropilmetil cellulose 0.3\%, Carbopol 980 [Noveon Europe, Brussels, Belgium], Dequest 2060S [Solutia, St. Louis, $\mathrm{MO}]$ ) and never came in direct contact with the corneal surface. A drop of antibiotic (ofloxacin $0.3 \%$ ) was instilled in the lower conjunctival fornix at the end of each examination, and the eye was reexamined at the slit lamp to verify the integrity of the corneal surface.

A scan of the full thickness of the cornea was automatically performed for each participant; the examination lasted 1.5 to 2.5 minutes.

Each scan recorded 350 images at a distance of $1.5 \mu \mathrm{m}$, on a $z$-axis, from one another. Each scan presented two to four complete passages from the endothelium to the superficial epithelium. The examinations were performed with a standard $40 \times$ object lens, and each photo frame included an area of approximately $440 \times 330 \mu \mathrm{m}$.

Image Analysis. The $z$-scan curve (a graphic showing the depth coordinate on the $z$-axis and the level of reflectivity on the $y$-axis $)^{9}$ of each scan was studied, and the images relative to the superficial and basal epithelium, the anterior and posterior stroma, and the subbasal plexus were selected.

All the areas of the $z$-scan curve where the superficial epithelium and endothelium peaks were clearly recognizable were considered. The depth values on the $z$-axis indicated by the software were used to determine corneal thickness.

The depth values indicated for the points in the $z$-scan corresponding to the first stroma photo frame after an image of an endothelium and the last stroma photo frame before an image referring to the basal epithelium were used to determine stromal thickness.
The corneal and stromal thicknesses taken into consideration for statistical analysis were obtained by calculating the mean score of all values taken from each $z$-scan curve.

Cell densities of the superficial and basal epithelium and apparent cell densities of the anterior and posterior stroma were evaluated. The images previously used to determine stromal thickness were taken into consideration in the study of the stroma.

In all cases cell density was determined through the manual cell counting procedure present in the software, taking into consideration the whole area marked as available for the cell count. The cells partially contained in the area analyzed were counted only along the right and lower margins. Results are expressed in cells per square millimeter.

The interobserver variation was calculated by comparing the values of cell density obtained by a second independent investigator using the same criteria. The image of the subbasal plexus where the highest number of nerve fibers recognizable was selected for each scan.

Three parameters were taken into consideration: the number of nerves, tortuosity, and fiber reflectivity. The last two parameters were evaluated according to grading $(0-4)$ performed by comparison with the reference images, according to the method proposed by OliveiraSoto and Efron ${ }^{10}$ (Figs. 1, 2).

\section{Statistical Analysis}

All the data are expressed as the average \pm SD. The normal distribution of variables was verified by the Kolmogorov-Smirnov test. The comparison between mean scores was made by using the $t$-test for each single sample, specifying the average value of $C$ as the test value in the SS versus C comparison and the average value of SSI to compare SSII versus SSI. The level of significance was set at $P<0.01$.

A two-factor ANOVA (factors: cell layer and disease state) with multiple comparisons was performed for the cell densities, with the
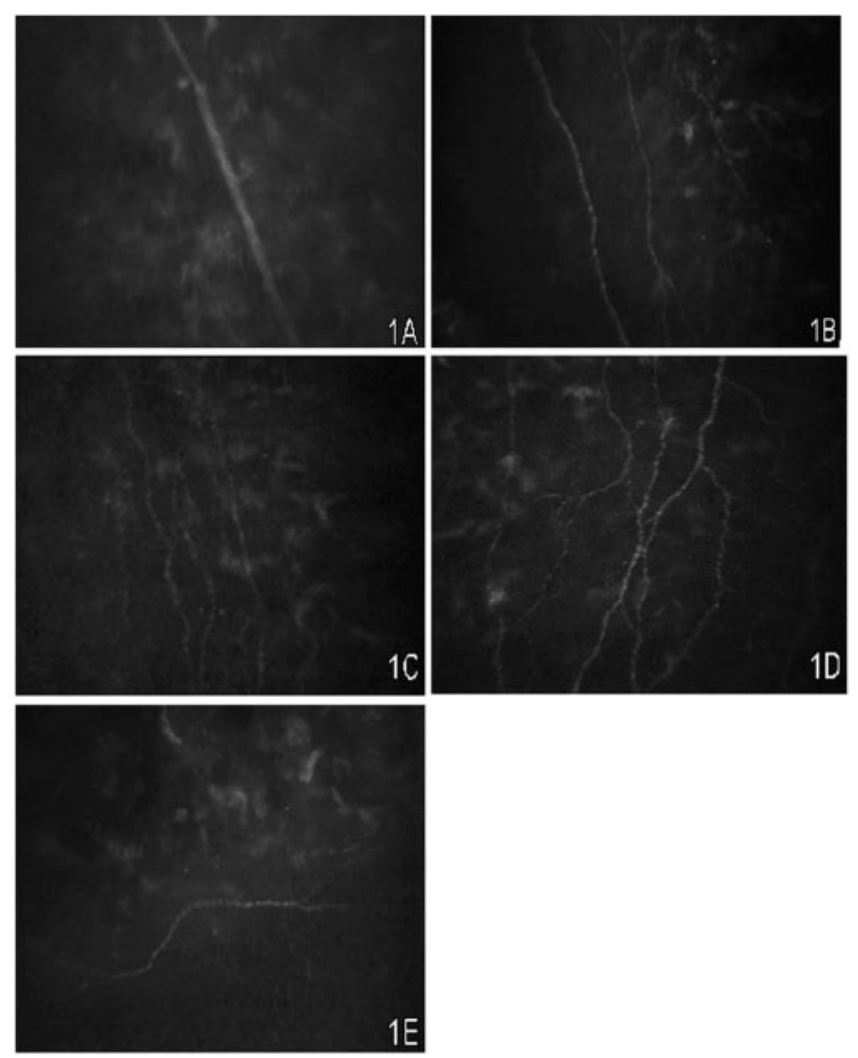

FIGURE 1. Grading scales for tortuosity provided by Oliveira-Soto and Efron $^{10}$ : Nerve fibers appear (A) grade 0, almost straight; (B) grade 1, slightly tortuous; (C) grade 2, moderately tortuous; (D) grade 3, quite tortuous; or (E) grade 4, very tortuous. 

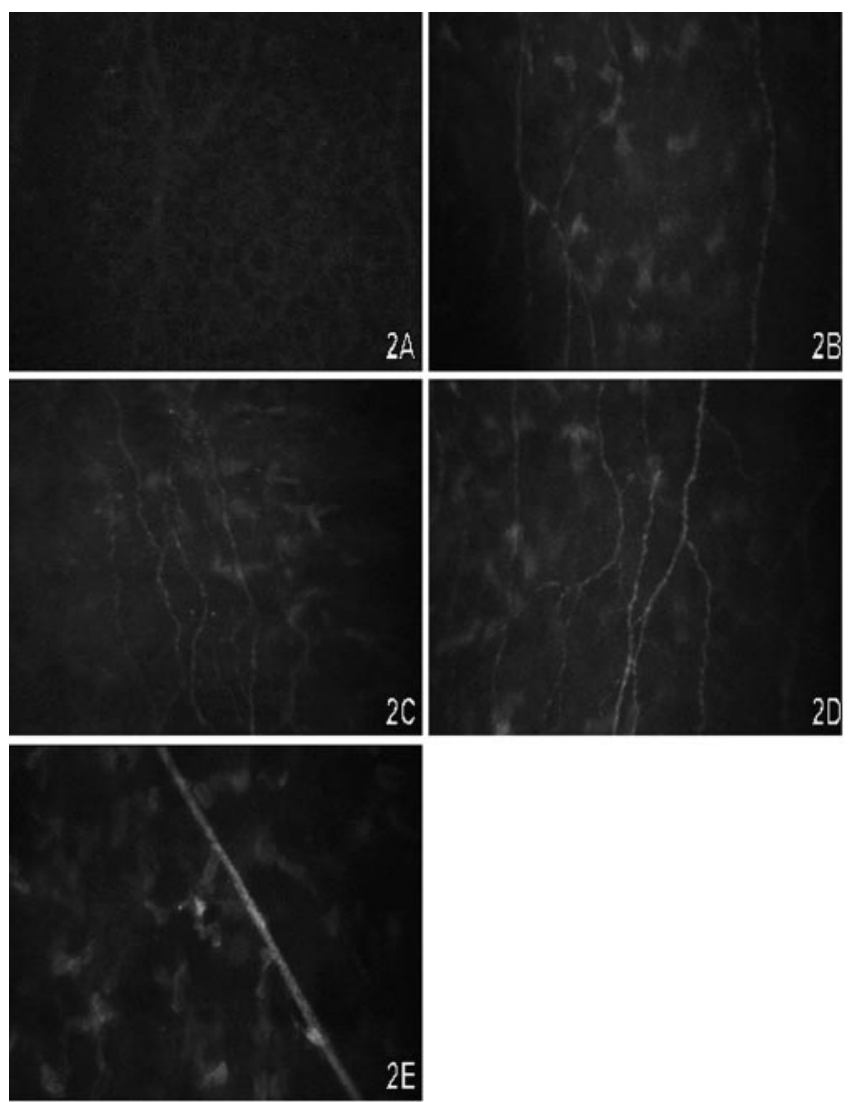

FIGURE 2. Grading scales for reflectivity provided by Oliveira-Soto and Efron $^{10}$ : Nerve fibers are (A) grade 0, almost indistinguishable from background; (B) grade 1, slightly dimmer than background; (C) grade 2 , comparable in reflectivity with background; (D) grade 3 , brighter than background; or (E) grade 4 , much higher in reflectivity than background.

least significant difference (LSD) post hoc test. The study of correlations between variables was performed by adopting the Spearman linear correlation index with confidence interval (CI) set at 99\%. The statistical analysis was performed with commercial software (SPSS for Windows, ver. 9.0; SPSS Sciences, Chicago, IL).

\section{Results}

\section{Clinical Data}

No relevant alterations were found in any participant after the confocal microscopic examination. The values relative to the clinical data (questionnaire, BUT, Schirmer I test, corneal sensitivity, IOP and fluorescein staining) for the SSI, SSII, and control groups are reported in Table 1 .
TABLe 2. Corneal and Stromal Thicknesses: Confocal Data

\begin{tabular}{lcc}
\hline & Corneal Thickness & Stromal Thickness \\
\hline SS & $514.74 \pm 19.85$ & $456.62 \pm 18.05$ \\
SSI & $512.33 \pm 19.91$ & $456.00 \pm 16.95$ \\
SSII & $516.55 \pm 20.13$ & $457.10 \pm 19.26$ \\
C & $550.00 \pm 21.46$ & $487.35 \pm 20.40$ \\
SS vs. C & $P<0.0001$ & $P<0.0001$ \\
SSI vs. SSII & $P=0.426$ & $P=0.805$ \\
& MDD* $^{*}=15.08$ & MDD $^{*}=8.55$ \\
\hline
\end{tabular}

Data are the mean \pm SD.

* Minimum detectable difference (in micrometers) between SSI and SSII ( $\alpha=0.01, \beta=0.20, n=15)$.

A statistically significant difference was found between SS and $\mathrm{C}$ in all the parameters studied $(P<0.0001, t$-test $)$. No statistically significant difference was found between the SSI and SSII groups in any of the parameters studied.

\section{Pachymetric Data}

Table 2 reports the data relative to corneal and stromal thickness in the SSI, SSII, and control groups. A statistically significant difference was found for SS compared to $\mathrm{C}$ regarding both the corneal and stromal thicknesses $(P<0.0001, t$-test $)$. Conversely, none of the two parameters showed a statistically significant difference between the SSI and SSII groups.

\section{Cellular Density Data}

The data relative to cellular density (in cells per square millimeter) for the SSI, SSII, and control groups are reported in Table 3. Comparisons between SS and C revealed a significant difference in the cell density of the superficial and basal epithelium $(P<0.001$, LSD) and in the apparent cell density of the anterior $(P<0.001, \mathrm{LSD})$ and posterior stroma $(P<0.01$, LSD). There were no statistically significant differences between the SSI and SSII groups in any of the parameters analyzed.

The interobserver variation was $5 \%, 2 \%, 8 \%$, and $6 \%$ for the superficial epithelium, basal epithelium, anterior stroma, and posterior stroma, respectively. A close correlation between the values obtained by the two investigators $(P<0.0001$, Pearson) was found in every cell layer.

\section{Subbasal Plexus Data}

Data regarding the number, tortuosity, and reflectivity of nerve fibers in the SSI, SSII, and control groups are indicated in Table 4. The comparison between SS and C showed a statistically significant difference in the number of nerve fibers visualized in the single photo frame $(P<0.0001$, t-test $)$ and in the tortuosity of fibers $(P<0.0001, t$-test $)$. The reflectivity of fibers did not record any statistically significant difference between the groups.

Table 1. Clinical Data

\begin{tabular}{|c|c|c|c|c|c|c|}
\hline & Questionnaire & BUT & Schirmer I & Sensivity & IOP (A) & Fluo \\
\hline SS & $2.52 \pm 0.52$ & $3.40 \pm 1.64$ & $5.43 \pm 2.49$ & $4.90 \pm 0.30$ & $14.88 \pm 1.05$ & $2.56 \pm 0.49$ \\
\hline SSI & $2.61 \pm 96.00$ & $3.00 \pm 1.41$ & $4.66 \pm 1.63$ & $4.90 \pm 0.29$ & $14.66 \pm 0.97$ & $2.58 \pm 0.41$ \\
\hline SSII & $2.46 \pm 0.53$ & $3.70 \pm 1.78$ & $6.00 \pm 2.88$ & $4.89 \pm 0.32$ & $15.05 \pm 1.01$ & $2.55 \pm 0.55$ \\
\hline $\mathrm{C}$ & $0.46 \pm 0.68$ & $11.55 \pm 1.90$ & $12.65 \pm 2.06$ & $5.61 \pm 0.38$ & $16.60 \pm 1.14$ & $0.14 \pm 0.18$ \\
\hline SS vs. C & $P<0.0001$ & $P<0.0001$ & $P<0.0001$ & $P<0.0001$ & $P<0.0001$ & $P<0.0001$ \\
\hline SSI vs. SSII & $P=0.278$ & $P=0.076$ & $P=0.007$ & $P=0.828$ & $P=0.15$ & $P=0.734$ \\
\hline
\end{tabular}

Data are the mean $\pm \mathrm{SD}$. 
TABLE 3. Cell Densities: Confocal Data

\begin{tabular}{lcccc}
\hline & Superficial Epithelium & Basal Epithelium & Anterior Stroma & Posterior Stroma \\
\hline SS & $985.05 \pm 107.56$ & $6197.37 \pm 180.34$ & $1263.45 \pm 80.83$ & $847.22 \pm 46.77$ \\
SSI & $965.40 \pm 96.00$ & $6261.13 \pm 168.36$ & $1274.93 \pm 32.69$ & $854.33 \pm 44.66$ \\
SSII & $999.80 \pm 115.67$ & $6149.55 \pm 178.03$ & $1255.00 \pm 103.47$ & $841.90 \pm 48.73$ \\
C & $1485.55 \pm 133.74$ & $5861.65 \pm 260.40$ & $1098.35 \pm 80.43$ & $797.85 \pm 41.97$ \\
$P$ & $<0.001^{*}$ & $<0.001^{* *}$ & $<0.001^{* * * *}$ & $<0.01^{* * * * * *}$ \\
\hline
\end{tabular}

Data are expressed as cells per square millimeter \pm SD.

$P$ is by ANOVA.

* SSI vs. C, SSII vs. C; $P<0.001$, LSD. SSI vs. SSII; $P=0.398$, LSD.

** SSI vs. C, SSII vs. C; $P<0.001$, LSD. SSI vs. SSII; $P=0.123$, LSD.

**** SSI vs. C, SSII vs. C; $P<0.001$, LSD. SSI vs. SSII; $P=0.475$, LSD.

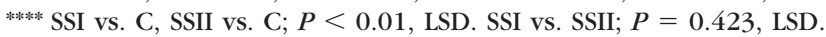

\# Superior epithelium. vs. basal epithelium, anterior stroma vs. basal stroma; $P<0.001$, LSD.

\#\# Superior epithelium vs. basal epithelium, anterior stroma vs. basal stroma; $P<0.001$, LSD.

\#\# Superior epithelium vs. basal eithelium, anterior stroma vs. basal stroma; $P<0.001$, LSD.

No statistically significant difference was found between the SSI and SSII groups. In SS group, a close correlation was noticed between BUT and Schirmer I result, and between Schirmer I result and fluorescein staining $(P<0.0001$, Spearman). The questionnaire results showed statistically significant correlations $(P<0.01$, Spearman) with fluorescein staining, Schirmer I result, and corneal sensitivity.

As far as the data obtained with the confocal microscope are concerned, a close correlation was found between superficial and basal epithelial cellular density and the number of nerve fibers per photo frame $(P<0.001$, Spearman) and between corneal and stromal thickness $(P<0.00001$, Spearman). Statistically significant correlations were also recorded between the following parameter pairs $(P<0.01$, Spearman): number of nerves and fiber tortuosity, fiber tortuosity and superficial epithelial density, superficial epithelial density and anterior stromal cellular density, and anterior stromal cellular density and corneal and stromal thickness.

In a comparison of the clinical data and those obtained with the confocal microscope, statistically significant correlations were found between questionnaire and nerve fiber tortuosity $(P<0.01$, Spearman $)$ and superficial epithelial cellular density $(P<0.0001$, Spearman), Schirmer I and number of nerves and superficial epithelial cellular density $(P<0.001$, Spearman), corneal sensitivity and fiber tortuosity $(P<0.01$, Spearman) and superficial epithelial cellular density $(P<0.01$, Spearman), and fluorescein staining evaluated according to the van Bijsterveld method and number of nerves $(P<0.01$, Spearman) and nerve tortuosity ( $P<0.01$, Spearman).

\section{Discussion}

During the past few years confocal microscopy has been used more and more frequently in the in vivo study of morphologic changes of the different corneal components in a wide range of pathologic processes. The analysis of data in the literature demonstrates that most studies performed so far in this field were qualitative studies based on the observation and description of the images of the various corneal structures.

This study offers a systematic evaluation of images, based on parameters that can be analyzed quantitatively. This approach permits a comparison between the characteristics and the extent of the morphologic changes found in sick patients and in the control group and, above all, highlights the relationships between clinical signs and morphologic changes in a statistically significant way.

The findings regarding the association of the questionnaire results with some of the other clinical parameters on the one hand and some of the confocal microscopy data on the other were surprising beyond expectations. The studies performed by Schein et al. ${ }^{11}$ and Hay et al. ${ }^{12}$ emphasized the weak link between the signs and symptoms that characterize dry eye syndrome. However, the results in the literature are rather heterogeneous-especially the characteristics of study samples and the type of questionnaire adopted. Nichols et al. ${ }^{13}$ recently reported data similar to those mentioned above, but using a questionnaire that evaluated symptom frequency exclusively and adopting a coefficient to adapt the data based on the use of artificial tears. In the same study, Nichols hypothesized that a correlation between signs and symptoms could be found only in the case of severe dry eye. The statistically significant correlation between symptoms and the results of the Schirmer test and between symptoms and rose bengal staining reported by Bjerrum ${ }^{14}$ in patients with SSI could be interpreted in the light of this. However, severe compromising of nerves and corneal sensitivity may impair the patients' perception of symptoms, as suggested by the inverted correlation between symptomatology and lissamine green staining $(r=$ $-0.431 ; P=0.011$, Spearman) observed by Adatia et al. ${ }^{15}$ Even when comparing the correlations within the individual groups, the analysis of our data does not permit the rating of variations of the associations between signs and symptoms based on the

TABLE 4. Number and Morphology of Subbasal Nerves: Confocal Data

\begin{tabular}{lccc}
\hline & Number of Nerves & Tortuosity (Grade, 0-4) & Reflectivity (Grade, 0-4) \\
\hline SS & $3.34 \pm 0.76$ & $2.62 \pm 0.94$ & $2.08 \pm 0.78$ \\
SSI & $3.33 \pm 0.81$ & $2.66 \pm 0.72$ & $2.13 \pm 0.83$ \\
SSII & $3.35 \pm 0.74$ & $2.60 \pm 1.09$ & $2.05 \pm 0.76$ \\
C & $5.10 \pm 0.79$ & $1.20 \pm 0.70$ & $1.85 \pm 0.87$ \\
SS vs. C & $P<0.0001$ & $P<0.0001$ & $P=0.083$ \\
SSI vs. SSII & $P=0.938$ & $P=0.727$ & $P=0.705$ \\
\hline
\end{tabular}

Data are the mean $\pm \mathrm{SD}$. 
severity of the pathologic situation. As a matter of fact, the correlation between the results of the questionnaire and the various parameters reaches the highest degree of significance in some cases in the participants in the SSI group (questionnaire with epithelial cellular density), in some cases in the participants in the SSII group (questionnaire with nerve fibers tortuosity), and in some cases in the control group (questionnaire with the Schirmer test and fluorescein staining).

The use of the confocal microscope enabled us to highlight a decrease in central corneal thickness and a significant reduction in stromal thickness in patients with Sjögren's syndrome I and II. The result being in line with those reported by Liu and Pflugfelder ${ }^{16}$ and by Tuominen et al. ${ }^{17}$

The hypothesis is that the corneal thinning noticed in those patients may be due to natural inflammatory processes. In particular, the hyperproduction of tumor necrosis factors TNF- $\alpha$ and interleukin IL-I would be responsible for apoptosis phenomena as well as for an increase in the proteolytic activity at the stroma level. However, the analysis of the average differences in corneal thickness $(-35.22, t$-test) and stromal thickness $(-30.46, t$-test) between SS and C groups showed that the reduction of stromal thickness in participants with SS is not sufficiently marked to justify the whole reduction of corneal thickness noticed in those patients. Such data, breaking away from those found by Tuominen et al., ${ }^{17}$ lead to the hypothesis that in patients with SS, the epithelium has equally reduced thickness, perhaps due to an increase in the forces tangentially acting on the ocular surface, given its abnormal irregularity.

IOP measurements in patients with SS gave lower significant results compared with the control group. This result may depend on the influence of the central corneal thickness on the results of the Goldmann applanation tonometry. The results of this study showed an average reduction in central corneal thickness by $35.22 \mu \mathrm{m}$ (approximately 6\%) and a reduced IOP average of $2.40 \mathrm{~mm} \mathrm{Hg}$ in the SSI+SSII group compared with the $\mathrm{C}$ group. Such data are in line with those reported in a recent meta-analysis by Doughty and Zaman ${ }^{18}$ and with what found by Tuonimen et al., ${ }^{17}$ who indicated an IOP difference of 2.5 and $2.8 \mathrm{~mm} \mathrm{Hg}$, respectively, for a difference in central corneal thickness of $10 \%$ and $6 \%$, respectively.

In the control group, we found apparent anterior and posterior stromal cell densities much higher than the values reported by Patel et al., ${ }^{19}$ Popper et al. ${ }^{20}$ and, for posterior stromal cells, by Prydal et al. ${ }^{21}$ Our results are similar to those reported by Mustonen et al. ${ }^{22}$ and Benitez del Castillo et al. ${ }^{23}$ These differences in quantification of the apparent stromal cell density (expressed in cells per square millimeter) are probably due to the use of more or less strict criteria for cell identification and count and to the different features of the instruments, lighting, and $z$-axis resolution in particular (optical sections include keratocyte nuclei of adjacent layers).

The reduced density of surface epithelial cells in patients with SS is connected to the corneal surface damage due to ocular dryness. Indeed, a significant correlation was found between the density of surface epithelial cells, the fluorescein staining and the results of tests made to evaluate secretion and the tear film (Schirmer I and BUT).

The basal epithelial cells and the anterior and posterior stromal cell components were shown to have increased density in patients with SS compared with the C group. The changes in the surface epithelium and the concomitant inflammatory processes act as a proliferating stimulus at basal level. The cellular changes at stromal level could be interpreted as a result of the inflammatory process that involves the ocular surface, determining a synchronous increase in the apoptotic phenomena at stromal level, in the proteolytic activity and proliferating stimuli.

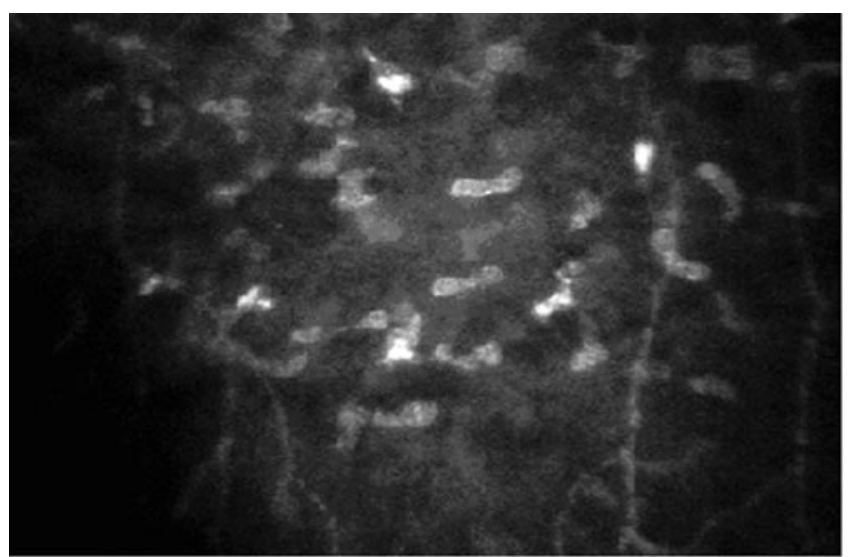

FigURE 3. Hyperreflective stromal cells

Furthermore, it is interesting to note that in many patients with SS the increase in stromal cellular density is accompanied by "activated keratocytes" (Fig. 3). Hyperreflective stromal cell nuclei images should be interpreted in a nonsingular way, as they could be representative of several cases of normal stromal cells in which the light ray of the confocal microscope strikes an unusual angle. However, we observed these images more frequently in patients with SS compared with the C group, as recently reported by Tuominen et al. ${ }^{17}$ and Benitez del Castillo et al. $^{23}$ It could be assumed that the findings correspond to keratocytes in a particular state of metabolic activation, induced by proinflammatory cytokines. Those cells would be able to secrete cell and neural growth factors (NGF), thus contributing to changes in the stromal and epithelial cells and nerves.

The number of nerve fibers in the subbasal plexus visible in the single photo frame was significantly lower in patients with SS compared with the C group. Tuominen et al., ${ }^{17}$ Benitez del Castillo et al. ${ }^{23}$ and Zhang et al. ${ }^{24}$ have recently performed this type of evaluation of the density of subbasal nerve fibers in SS dry eye, with conflicting results. However, it is interesting to note how the reduced number and increased tortuosity of the subbasal fibers that this study showed in patients with SS can be linked to the phenomena of corneal hypoesthesia typical of this disease and the consequences for tear secretion. The increased tortuosity of fibers may be due to the simultaneous action of detrimental phenomena and growth factors (NGF) secreted over the course of the inflammatory process.

The changes in the subbasal plexus fibers have an important effect on the complex regulation system of the ocular surface morphofunctional unit and can compromise various aspects of it, such as blinking, the tear reflex and trophism of the epithelial cells, thus neatly contributing to the increase of the vicious cycle of hypotearing-phlogosis- cell-nerve damage.

In conclusion, confocal microscopy permitted us to find several changes in the corneal morphology of patients affected by Sjögren's syndrome. We observed alterations in corneal thickness, cells, and nerves. On the basis of our results, we cannot exactly decode all the cause- effect relations among the different alterations of the ocular surface, which appears as a complex morphofunctional unit in which each variable interacts in different ways and at different times with many other variables. Several stages of the vicious cycle that characterizes the dry eye associated with Sjögren's syndrome are worth thorough study, to understand exactly its complex pathogenesis and the potential of therapeutic approaches for the control of the inflammatory process and for neuroprotection. 


\section{References}

1. Fox RI, Stern M, Michelson P. Update in Sjögren syndrome. Curr Opin Rheum. 2000;12:391-398.

2. Fox RI, Stern M. Sjögren syndrome: mechanisms of pathogenesis involve interaction of immune and neurosecretory systems. Scand J Rheumatol Suppl. 2002;116:3-13.

3. Borchers AT, Naguwa SM, Keen CL, et al. Immunopathogenesis of Sjögren's syndrome. Clin Rev Allergy Immunol. 2003;25:89-104.

4. Stern ME, Beuerman RW, Fox RI, Gao J, Mircheff AK, Pflugfelder SC. The pathology of dry eye: the interaction between the ocular surface and lacrimal glands. Cornea. 1998;17:584-589.

5. Stern ME, Gao J, Siemasko KF, et al. The role of the lacrimal functional unit in the pathophysiology of dry eye. Exp Eye Res. 2004;78:409-416.

6. Rolando M. Sjögren's syndrome as seen by an ophthalmologist. Scand J Rheumatol Suppl. 2001;115:27-31.

7. Vitali C, Bombardieri S, Jonsson R, et al. Classification criteria for Sjögren's syndrome: a revised version of the European criteria proposed by the American-European Consensus Group. Ann Rheum Dis. 2002;61:554-558.

8. Schiffman RM, Christianson MD, Jacobsen G, Hirsch JD, Reis BL. Reliability and validity of the Ocular Surface Disease Index. Arch Ophthalmol. 2000;118:615-621.

9. Mastropasqua L, Nubile M. Basic principles of confocal microscopy of the cornea. In: Confocal Microscopy of the Cornea. Thorofare, NJ: SLACK Inc.; 2002:3-4.

10. Oliveira-Soto L, Efron N. Morphology of corneal nerves using confocal microscopy. Cornea. 2001;20:374-384.

11. Schein OD, Tielsch JM, Munoz B, Bandeen-oche K, West S. Relation between signs and symptoms of dry eye in the elderly: a populationbased perspective. Ophthalmology. 1997;104:1395-1401.

12. Hay EM, Thomas E, Pal B, Hajeer A, Chambers H, Silman AJ. Weak association between subjective symptoms or and objective testing for dry eyes and dry mouth: results from a population based study. Ann Rheum Dis. 1998;57:20-24.

13. Nichols KK, Nichols JJ, Mitchell GL. The lack of association between signs and symptoms in patients with dry eye disease. Cornea. 2004;23:762-770.
14. Bjerrum KB. Test and symptoms in keratoconjunctivitis sicca and their correlation. Acta Ophthalmol Scand. 1996;74:436-441.

15. Adatia FA, Michaeli-Cohen A, Naor J, Caffery B, Bookman A, Slomovic A. Correlation between corneal sensitivity, subjective dry eye symptoms and corneal staining in Sjögren's syndrome. Can J Ophthalmol. 2004;39:767-771.

16. Liu Z, Pflugfelder SC. Corneal thickness is reduced in dry eye. Cornea. 1999; 18:403-407.

17. Tuominen IS, Konttinen YT, Vesaluoma MH, Moilanen JA, Helinto M, Tervo TM. Corneal innervation and morphology in primary Sjögren's syndrome. Invest Ophthalmol Vis Sci. 2003;44:25452549.

18. Doughty MJ, Zaman ML. Human corneal thickness and its impact on intraocular pressure measures: a review and meta-analysis approach. Surv Ophthalmol. 2000;44:367-408.

19. Patel SV, McLaren JW, Hodge DO, Bourne WM. Normal human keratocyte density and corneal thickness measurement by using confocal microscopy in vivo. Invest Ophthalmol Vis Sci. 2001;42: 333-339.

20. Popper M, Morgado AM, Quadrado MJ, Van Best JA. Corneal cell density measurement in vivo by scanning slit confocal microscopy: method and validation. Ophthalmic Res. 2004;36: $270-276$.

21. Prydal JI, Franc F, Dilly PN, Kerr Muir MG, Corbett MC, Marshall J. Keratocyte density and size in conscious humans by digital image analysis of confocal images. Eye. 1998;12:337-342.

22. Mustonen RK, McDonald MB, Srivannaboon S, Tan AL, Doubrava MW, Kim CK. Normal human corneal cell populations evaluated by in vivo scanning slit confocal microscopy. Cornea. 1998;17: 485- 492 .

23. Benitez del Castillo JM, Wasfy MAS, Fernandez C, Garcia-Sanchez J. An in vivo confocal masked study on corneal epithelium and subbasal nerves in patients with dry eye. Invest Ophthalmol Vis Sci. 2004;45:3030-3035.

24. Zhang M, Chen J, Luo L, Xiao Q, Sun M, Liu Z. Altered corneal nerves in aqueous tear deficiency viewed by in vivo confocal microscopy. Cornea. 2005;24:818-824. 